This item was submitted to Loughborough's Research Repository by the author.

Items in Figshare are protected by copyright, with all rights reserved, unless otherwise indicated.

\title{
Exploring the potential of salivary and blood immune biomarkers to elucidate physical frailty in institutionalized older women
}

PLEASE CITE THE PUBLISHED VERSION

https://doi.org/10.1016/j.exger.2019.110759

\section{PUBLISHER}

Elsevier

VERSION

AM (Accepted Manuscript)

\section{PUBLISHER STATEMENT}

This paper was accepted for publication in the journal Experimental Gerontology and the definitive published version is available at https://doi.org/10.1016/j.exger.2019.110759

LICENCE

CC BY-NC-ND 4.0

\section{REPOSITORY RECORD}

Furtado, Guilherme Eustáquio, Matheus Uba Chupel, Luciele Minuzzi, Miguel Patrício, Marisa Loureiro, Stephan Bandelow, Eef Hogervorst, José Pedro Ferreira, and Ana Maria Teixeira. 2019. "Exploring the Potential of Salivary and Blood Immune Biomarkers to Elucidate Physical Frailty in Institutionalized Older Women". Loughborough University. https://hdl.handle.net/2134/12659684.v1. 


\title{
Exploring the potential of salivary and blood immune profile makers to explain physical frailty in institutionalized older women
}

\begin{abstract}
Identification of older populations at increased risk of physical frailty using biochemical approaches could improve screening accuracy. The aim of this study was to study the relationship between immune markers and independent components of physical frailty in institutionalized older women. A sample of 139 institutionalized-dwelling women, aged 75 years and older, were assessed for biosocial factors and general health status, pro and anti-inflammatory cytokines, sex steroid hormones, salivary antimicrobial proteins, blood cells counts and the five Fried's physical frailty components that allowed for classification of the sample into frail, prefrailty and not-frail subgroups. Results showed that cytokines IL-6, IL-10, IL-1 $\beta, \mathrm{TNF}-\alpha$, and the TNF- $\alpha / \mathrm{IL}-10$ ratio, mean corpuscular haemoglobin, salivary cortisol and $\alpha$-amylase were all associated with frailty. Weakness and Exhaustion were the frailty components that were most strongly associated with these biomarkers. Salivary $\alpha$-amylase was the biomarker that best explained frailty, as it was associated with all five components of physical frailty, and could be used as a potential screening tool. Future research needs to investigate the causal-effect association between salivary innate immune makers, susceptibility to infection and frailty.
\end{abstract}

Key words: frail older adults, salivary biomarkers, inflammatory cytokines, sex steroids hormones, neuroendocrine, weakness, exhaustion 


\section{Introduction}

Biomarkers reflecting organ system functioning are intrinsically inter-related and are well suited for the study of the development of frailty (Gale et al., 2013; Walston, 2002). Especially in advanced age, the clinical health status is characterised by long-term musculoskeletal, immune and neurocognitive morbidities, that impact on people's physical functioning. Clinical health outcomes are more than just the absence of disease, and include all physiological structures related to the aging process. Recently, studies have revealed that poor clinical health is associated with an imbalance in several biomarkers involved in the immune system, especially in frail individuals (Gale et al., 2013; Kumar et al., 2014; Leng et al., 2011, 2007; Lu et al., 2016).

In the last decade, frailty was conceptualized as a state in which reserve function across multiple physiologic systems declines, compromising the individual's capacity to withstand biological stress, thereby predisposing them to poor health, functional decline, institutionalization and death (Mitnitski et al., 2015; Rockwood and Bergman, 2012). Recent approaches to the frailty syndrome include factors connected with multiple negative psychosocial and emotional facets that manifest during the aging process and where frailty has a negative effect on psychological aspects (Freitag and Schmidt, 2016). Using a classical approach, Linda Fried and colleagues established the Physical Frailty (PF) concept and identified five components, Weakness, Slowness, Exhaustion, Weight loss and Low levels of Physical Activity (PA) to characterize it (Fried et al., 2001). With this protocol, it is possible to categorise the older population in frail, pre-frail and non-frail subgroups, according to the presence or absence of each of the five individual components (see for a detailed description, the methodology section). Accumulating evidence from observational studies also supports a progressive relationship between the frail syndrome and the malfunctioning of the immune and neuroendocrine systems (Clegg et al., 2013).

A study showed that a decrease in the immune response is closely associated with several aspects of frailty, including sarcopenia and osteopenia (Aw et al., 2007). The molecular mechanisms 
involved in this relationship are quite complex, which hinders firm conclusions about which biomarkers could have a greater power in explaining the early development of the frail phenotype (Yao et al., 2011). Some experts suggest that the main reason for the difficulty in understanding the phenomenon is the diversity of mechanisms and players involved in the immune system, such as catecholamines, sex steroid hormones, anti and pro-inflammatory cytokines, and several cellular subsets that impact on immune function (Calvani et al., 2015; Darvin et al., 2014; Mitnitski et al., 2015). Systematic reviews and meta-analyses showed that frailty (and pre-frailty) were associated with higher levels of interleukins (IL). Several studies reported that frailty in older community dwelling or institutionalized dwelling persons was strongly associated with high levels of pro-inflammatory markers (Baylis et al., 2013; Lu et al., 2016) (Soysal et al., 2016a). A recent paper (Rossi et al., 2019) also highlighted the importance of using the ratio between anti and pro-inflammatory markers when seeking associations with several health conditions associated with aging, such as the TNF- $\alpha / \mathrm{Il}-10$ ratio, which has been used as an inflammatory index (Chupel et al., 2017).

Numerous researchers have sought to assess the most prominent clinical or physiologically relevant biomarkers and their relationship with PF and poor clinical status, or assessed how putative predictors of PF could serve as additional screening tools (Schoufour et al., 2016). The Newcastle 85+ Study assessed whether a set of 40 biochemical markers predicted the frailty index, and examined their collective effect in predicting mortality as compared with individual biomarkers (Mitnitski et al., 2015). The results showed that the combined biomarkers Frailty-index was more powerful for mortality prediction than any individual biomarker. In another study, serum sirtuins (NAD dependent deacetylases with anti-ageing properties) were described as promising non-invasive biomarkers for the PF status (Kumar et al., 2014). Associations between PF in older institutionalized men and levels of inflammatory makers, in particular IL-6, Tumour Necrosis Factor-Alpha (TNF- $\alpha$ ) and high sensitivity CRP have also been reported (Hubbard et al., 2009; Soysal et al., 2016a).

Analysing multisystem risk factors of frailty in cross-sectional data of 1685 older adults aged 
$\geq 55$ in the Singapore Longitudinal Ageing Studies, the researchers found that white blood cell counts, haemoglobin, albumin, lymphocytes and total cholesterol were also highly associated with pre-frail and frail subgroups (Ng et al., 2014). A recent study that focused on the female population found that high concentrations of C-Reactive Protein (CRP) were more strongly predictive of frailty incidence in women than in men (Gale et al., 2013). Many studies in the scientific literature are based on community-dwelling elderly people, and the extrapolation of these results to a sample of institutionalized older people can be imprecise.

Since institutionalization increases demands on public health systems, especially on medical and long-term social services, the research interest for this subpopulation has grown in the last years (Conroy et al., 2011). In studies conducted with samples of elderly who require social support and health care, the incidence of the frailty (15\% to $40 \%)$ trait seems to reach higher percentages in women, but with men dying earlier (Abizanda et al., 2014; Furtado et al., 2018; González-Vaca et al., 2014). In Portugal, the results of the first study recently published indicated that the prevalence of PF in elderly communities was $34.9 \%(n=339$ participants $)$ and the incidence among older women $(40.9 \%)$, higher than that in older men (32.5\%) (Duarte and Paúl, 2015). To our knowledge, this is the first study looking at the association between PF (physical frailty) and biochemical parameters in sample of Portuguese institutionalized elderly.

Although cognitive, psychosocial and/or functional impairment (including physical frailty) are the main predictors of frailty that lead to elderly institutionalized long-term living (Luppa et al., 2009), the study of blood and salivary biomarkers could give important clues to the nature of the underlying causal deficits leading to age-related frailty, thereby helping to expose targets for early preventative interventions (Mitnitski et al., 2015). In addition, to understand how some biochemical markers independently associate with different PF components could help tailor the interventions that aim to prevent, mitigate and even reverse the incidence of $\mathrm{PF}$.

Older adults living in institutionalized care are a dissimilar population with regards to many 
geriatric clinical health outcomes. Many questions remain unresolved for this population, including how some of the biomarkers are related with PF and their components. To our knowledge, the use of salivary immune and hormonal markers and their association with PF has not been reported in the literature. In this study, we propose to assess several biomarkers (cytokines, sex steroid hormones, anti-microbial proteins and blood counts) reflecting different organ systems' functioning may explain PF and test the strength of their relationship with Fried's five components of PF in institutionalized older women. It was hypothesized that the frail sub-group would have a lower immune profile than the prefrail and non-frail groups, and that both salivary and blood biomarkers could also reveal significant associations with frailty in this population which would be useful for screening.

\section{Material and Methods}

\subsection{Design}

This is a cross-sectional exploratory study of institutionalized-dwelling individuals based on a survey aimed to assess the PF incidence in people living (long-term) in centres for social and health care support (CHS). The sample consisted of a subset of the participants who took part in the previous published study protocol (Teixeira et al., 2016). Cross-sectional data from the baseline assessments were used for the current paper.

\subsection{Eligibility criteria}

Participants were women, aged 75 years and over, who were willing to take part in the study and who were under medical supervision with updated and monitored prescribed drugs. The specific criteria for participant's exclusion were: the presence of any type of health condition that could prevent testing of autonomy, such as severe cardiomyopathy, uncontrolled cardiorespiratory illness and any musculoskeletal conditions that might prevent testing (i.e. recent fractures); having a diagnosed mental health disorder, severe cognitive impairment (SCI) or severe dementia (the Mini-Mental State 
Examintion was applied and scores below 11 points were considered abnormal and used for SCI and dementia screening); diagnosed hearing and vision impairment, morbid obesity (IMC $\geq 40$ ); having no controlled and updated drug therapy or the use of medications that could cause attentional impairments and affect motor activity (e.g. anxiolytics and anti-depressants).

\subsection{Sample selection}

The visits to all CHS were held between March 2016 and November 2017, and a total of five institutions with similar conditions were selected, in order to reduce bias in the final study sample. All selected CHS had an average of 85 residents, with different degrees of incapacity. Each institution had a daycentre and nursing support for clinical stabilization of acute diseases, and had similar daily routines and organization, including health and social care. The health staff in all CHS were composed of a principal and several assistant nurses, a medical general practitioner, a social worker, physiotherapists, occupational therapists and a nutritionist.

The first stage for the participants' assessment was information provided by the medical professionals from each CHS on potential participants, combined with information for clinical primary outcomes. A convenience sampling assignment was used. The initial recruitment consisted of $\mathrm{n}=486$ $(100 \%)$ institutionalized-dwelling potential participants. Before the initial screening, $\mathrm{n}=128$ individuals (25\%) were excluded because of several negative clinical health conditions, such as severe physical, cognitive, hearing or visual impairment and other specific impairments, inconsistent data and drop-out during the data collection phase. The final analysed sample consisted of $n=358$ older women.

\subsection{Ethical statement}

All the CHS directors and potential participants who expressed interest in participating in the study signed an informed consent form, in which the privacy and anonymous identity of the data collected were guaranteed and any required access to the participants' medical records was given. The 
study protocol was approved by the Multidisciplinary Ethical Committee and Review Board of the Faculty of Sport Sciences and Physical Education, University of Coimbra [reference code: CE/FCDEF-UC/000202013], respecting the Portuguese Resolution [Article. 4st; Law number. $12 / 2005,1$ st series)] on ethics in research (Braga, 2013), and complied with the guidelines of the Helsinki Declaration for human research (Petrini, 2014).

\subsection{Outcome measures}

Data collection of all variables was organized by the principal investigator and was performed by independent trained specialists of the research team. The same evaluators for each study domain performed the data collection in all study participants. The biosocial and general health indicators were selected as covariates, following the recommendations established in the original and current studies (Abizanda et al., 2013; Fried et al., 2001). However, only the biosocial and general health indicators which had shown statistical differences between frail sub-groups in the comparison analysis were treated as covariates in the subsequent correlational statistical analyses. As such, age, education level (in years), morbidity index (in number) and Mini-Mental State Examination scores were selected as co-variables, all introduced in the correlation and regression models and analysed as continuous variables. Other data of biosocial and general health status analysed that were part of the study but did not present significant differences were not entered as covariate in the adjusted models.

\subsubsection{Physical Frailty screening}

The Fried's Frailty Phenotype construct is operationalized using five criteria that divide the population in non-frailty, pre-frailty and frailty older individuals. The prevalence and the presence of each one of the five Fried's PF independent components was assessed. A positive evaluation in one or two criteria classified the participants as pre-frail, in three or more as frail and as non-frail when the 
subject had none of the five PF independent components, thus establishing a categorical classification (Fried et al., 2001).

The following five components of PF were assessed: Weight loss was assessed by self-report of unintentional weight loss of four kilograms or more in the last six months, validated by medical records. Poor endurance and energy (self-reported exhaustion) was evaluated by negative concordance of question number 7 ("I felt that everything I did was an effort") and 20 ("I could not get "going"), of the Center of Epidemiologic Studies for Depression questionnaire (CES-D), which in the original study had shown a significant association with a direct measurement of maximum rate of oxygen consumption $\left(\mathrm{VO}_{2 \max }\right)$, and was predictive of cardiovascular disease. Weakness was analysed using the handgrip strength test (HGT). This test uses a hand-held dynamometer [Lafayette Dynamometer, model 78010, United States] and strength is measured in kilograms. The subject holds the dynamometer in the hand to be tested, with the elbow by the side of the body. When ready, the subject squeezes the dynamometer with maximum isometric effort, which is maintained for 5 seconds. The best result of the two trials was used for scoring purposes. Participants who were unable to perform the HGT and those in the lowest $20 \%$ were categorized as positive. The cutoff values for HGT of $\geq$ $29 \mathrm{~kg}$ for male and $\geq 17 \mathrm{Kg}$ for female were adopted (Fried et al., 2001). Slowness was measured by the '15 feet (4,6 meters) walking test', adjusted for gender and height. Based on cut off values of Fried's study population, times of $\geq 7$ seconds for males and $\geq 6$ seconds for females were adopted for positive scores of slowness. The best time of the two trials was used for final scoring (Fried et al., 2001). Low Physical Activity (PA) levels were assessed by the International PA Questionnaire short version (IPAQ-SV). The IPAQ-SV asks about three specific types of activity undertaken and time being sedentary. The specific types of activity assessed are walking, moderate-intensity activities and vigorous intensity activities. Frequency (measured in days per week) and duration (time per day) are collected separately for each specific type of activity. The total volume and the number of day/sessions was included in the IPAQ-SV analysis. There are three levels of PA suggested for classification: 
inactive, minimally active and highly active. Participants classified as inactive had a positive score for this PF component.

\subsubsection{Biomarkers analysis}

All saliva and blood sample collections for the biomarkers analysis occurred in the morning (between 10:00h - 12:00h). Saliva samples were collected by passive drool, that consisted of the participant allowing saliva to collect on the floor of the mouth, then to lean forward and dribble into a polypropylene tube for 3 minutes. Prior to the saliva collection, subjects were asked to rinse their mouth with water to remove any food residues $20 \mathrm{~min}$ before sample collection. Saliva samples volumes were measured, aliquoted and stored at $-20^{\circ} \mathrm{C}$ until further analysis. Salivary concentrations of testosterone (TT), cortisol (COR), dehydroepiandrosterone (DHEA) and immunoglobulin-A (Ig-A) were analysed by ELISA (Salimetrics UK, 2017). Salivary concentrations of lysozyme (LYS), IL-1 $\beta$ and IL-6 were also analysed by standard ELISA (Salimetrics UK, 2017). Salivary alpha-Amylase ( $\alpha$ Amylase) was analysed using a kinetic assay (Salimetrics UK, 2017). Blood samples were collected by venepuncture by a registered nurse, and were allocated into $\mathrm{k}_{3}$ EDTA and serum tubes. Determination of blood counts was done immediately after blood collection, using an automated haematology analyser by Coulter AcT Diff, Beckman Coulter, USA. The remaining blood was separated by centrifugation and the plasma and serum samples were kept frozen at $-80^{\circ} \mathrm{C}$ until further use. Plasma levels of TNF- $\alpha$, IFN- $\gamma$ and IL-10 were analysed by ELISA analyser Invitrogen ${ }^{\circledR}$, CA. Additionally, the ratio of TNF- $\alpha / \mathrm{IL}-10$ was calculated to assess the pro/anti-inflammatory balance, according to previous studies (Chupel et al., 2017; Rossi et al., 2019). Serum CRP was determined using the Horiba Medical Pentra C200 [Kyoto, Japan].

\subsubsection{Biosocial status}


Sociodemographic variables of chronological age and level of education were also collected. The level of education were classified according to the Portuguese educational system (Fernandes, 2007), and refers to the number of years spent in school by the participants (entered as a continuous variable). Anthropometric measures included body mass, determined using a portable scale (Seca ${ }^{\circledR}$, model 770, Germany) with a precision of 0.1 kilograms; stature was determined using a portable stadiometer (Seca Body meter ${ }^{\circledR}$, model 208 , Germany) with a precision of 0.1 centimeters. Body mass index (BMI) was calculated according to the formula $\left[\mathrm{BMI}=\right.$ weight $/$ height $\left.^{2}\right]$ and analysed as a continuous variable. The standardized procedures described by Lohan and colleagues were followed for the collection of anthropometric data (Chumlea, Baumgartner, 1989).

\subsubsection{General health status screen}

Nutritional status was measured using the Mini Nutritional Assessment (MNA) that consists of 18 questions. The maximum score is 30 points (pts), and classifies subjects as well-nourished (24 to $30 \mathrm{pts}$ ), at risk of malnutrition (17 to $23.5 \mathrm{pts)}$ or as malnourished when scoring 17 points and lower (Guigoz, 2006). The Charlson Comorbidity Index (CCI) measures burden of disease and has a weighted index based on 19 comorbid conditions. This score can be combined/adjusted with age and gender to form a single index (0-10 points) to be used as a continuous variable (Charlson et al., 1994). Daily medications used (DMU) by the participants were assessed through question number six of the MNA, that asks the participant if he/she takes more than 3 or less prescription drugs per day (entered as a continuous variable). In addition, both CCI and DMU were checked via the medical records provided by the medical staff. The MMSE was used to assess cognitive profile. This test assesses five areas of cognition (Folstein et al., 1975). The scores range from 0 to 30 points, and can be evaluated as a continuous variable. Categorically, the scores between 19 to 24 points are used for screening mild cognitive impairment and below 11 is considered severe. 


\subsection{Statistical analysis}

The assumption of normality was checked by using Shapiro Wilk tests and visual inspection of plots. Continuous data are described by their medians, $1^{\text {st }}$ and $3^{\text {th }}$ quartiles, whereas qualitative data are described by absolute and relative frequencies. The comparison of continuous variables between the frailty sub-groups (van der Ploeg et al., 2014) was performed using ANOVA or Kruskal-Wallis, depending of normality distributions of data. The PF components of Exhaustion and Weight loss (used as binary categorical variables) and their association with biomarkers was assessed through logistic regression. For this purpose, the odds ratio $(\mathrm{OR})$ reference value was used. Odds ratios greater than 1 indicate positive variations in the independent variable and values less than 1 reflect negative (or inverse) variations. To estimate the variation in relative terms (percentages), the formula: OR - 1 x 100 was used (van der Ploeg et al., 2014). The association between PF components of Slowness, Weakness and low levels of PA (all used as continuous variables) and biomarkers were investigated using linear regression. To report the variation in relative terms (percentages), we reported adjusted $\mathrm{R}^{2}$ values. First, univariate logistic regression analyses were used to describe the unadjusted effect. This model simply included one dependent variable and one independent variable. Second, multivariate analysis was run to analyse the same association in the adjusted model 2 (using age, nursing homes, educational level, comorbidities, and cognitive state as co-variates). The magnitude of associations was classified following recommendations: trivial $(\mathrm{r}<0.1)$; small ( $\mathrm{r}$ from 0.1 to 0.3$)$; moderate ( $\mathrm{r}$ from 0.3 to 0.5 ); strong ( $\mathrm{r}$ from 0.5 to 0.7 ) and robust (r from 0.7 to 0.9) (Batterham and Hopkins, 2006). Biochemical markers were assumed as independent variables and PF components were dependent in these regression analysis. All statistical tests were performed at the 0.05 level and $95 \%$ confidence intervals were given. IBM SPSS Statistics 21.0 and in R 3.3.1 software were used for all computations.

\subsection{Results}


Table 1 presents the biosocial characteristics of the complete sample and the results from the frail subgroups analysis. Of all participants, 144 (39.2\%) were considered as frail, 136 as pre-frail $(38,1 \%)$ and 78 were classified as non-frail $(22.3 \%)$. When frail sub-groups were compared for biosocial and global health status (co-variates), statistical differences were found for level of education $(p=0.030)$, stature $(p<0.001)$, comorbidities $(p=0.006)$, depression $(p=0.009)$ and cognitive status $(\mathrm{p}<0.001)$.

\section{[Insert table 01 about here, please]}

The biomarkers levels for the complete sample and results by frail subgroup are presented in table 2. Statistical differences were found for levels of salivary IL-6 $(\mathrm{p}<0.001)$, IL-1 $\beta(\mathrm{p}=0.01)$, $\operatorname{COR}(\mathrm{p}<0.001)$ and $\alpha$-amylase $(\mathrm{p}=0.02)$, as well as plasma IL-10 $(\mathrm{p}=0.049)$, TNF- $\alpha(\mathrm{p}=0.035)$ and in the TNF- $\alpha /$ IL-10 ratio $(p<0.001)$. Differences in mean corpuscular haemoglobin $(\mathrm{MCH})(\mathrm{p}=$ 0.013 ) were also found between groups.

\section{[Insert table 02 about here, please]}

In the second step, Spearman and partial correlations were used to analyse the correlations among biochemical makers and PF total score. Total score of PF was statistically significantly associated with all biomarkers $(\mathrm{p}<0.05)$. The results indicated a negative correlation with IL- $6, \alpha-$ amylase and MCH. Positive correlations of IL-10, TNF- $\alpha$ and IL-1 $\beta$ were shown $(p<0.05)$. After controlling for co-variates all correlations remained stable, expect for IL-1 $\beta$, TNF- $\alpha$ and the TNF$\alpha /$ IL-10 ratio (Table 3). 
On the basis of this outcomes, regression analysis was used to explore the relationship between each biomarker (assuming these as dependent variables), and the PF components (independent variables) in this third step. The results of table 3 show that in the unadjusted and adjusted (for age, nursing home, CCI, MMSE and education levels) models, salivary IL-6, IL-1 $\beta$ and $\alpha$-amylase makers were associated with the PF component Weakness. Plasma IL-10, TNF- $\alpha$, MCH and salivary $\alpha-$ amylase, COR and IL-1 $\beta$ showed an association with Self-reported Exhaustion, although $\alpha$-amylase presented a significant relationship only after adjusting the model for covariates $(\beta=.003, \mathrm{OR}=.998$, $95 \% \mathrm{CI}=[.997 ; 1003], \mathrm{p}=.048)$. Only salivary $\alpha$-amylase presented a significant association with the independent PF component of Low Levels of PA, in both adjusted $\left(r^{2}=.044, \beta=.002, p=.008\right)$ and unadjusted $\left(\mathrm{r}^{2}=.033, \beta=.003, \mathrm{p}=.009\right)$ regression models. The TNF- $\alpha / \mathrm{IL}-10$ ratio presented significant associations with all PF components (considering adjusted models), except for nonintentional Weight Loss.

\subsection{Discussion}

Analysing the associations between the biomarkers and PF in institutionalized older women, results indicated that salivary levels of IL-6, IL-1 $\beta$, COR, $\alpha$-amylase and plasma levels of IL-10, TNF$\alpha, \mathrm{MCH}$ and the TNF- $\alpha / \mathrm{IL}-10$ ratio were different for the PF subgroups. Exploring the relationship between the individual PF components and salivary IL-6, IL-1 $\beta$, COR $\alpha$-amylase and plasma TNF- $\alpha$, results showed that mucosal immune makers ( $\alpha$-amylase and COR), pro-inflammatory (IL-6, IL-1 $\beta$ and TNF- $\alpha$ ) and anti-inflammatory (IL-10) cytokines as well as the TNF- $\alpha /$ IL-10 ratio showed a significant relationship with Weakness and Exhaustion.

The human immune system is a host defence system involving many biological structures within an organism that defends against several types of pathogens or other substances from the environment, neutralizing viruses, bacteria and fungi through mechanisms such as phagocytosis (Licastro et al., 2005). All biomarkers involved in this study have protective functions directly or 
indirectly influencing the immune response, its efficacy decreasing with advancing age. Studies suggest that there are abnormal increased immunosenescence processes in frail individuals, particularly in pro-inflammatory cytokines such as IL-6 and IL-1 $\beta$ (Soysal et al., 2016b). High levels of these cytokines in older people have been associated with neurodegenerative, cardiometabolic, and musculoskeletal disorders (Licastro et al., 2005). However, our study showed unexpected lower values of IL-6 in the frail subgroup, contradicting the results of other findings (Leng et al., 2007).

A likely explanation for these results was presented by Maggio and colleagues, who explained the causal role of lower levels of IL-6 (and other cytokines such as IL-12 and IL-23) in very old frail people (as was the case for our study sample), with possible very high decreases in the immune response, involving the Pattern Recognition Receptors-PRR (Maggio et al., 2006). PRRs recognize varied pathogen by their molecular patterns, leading to the secretion of inflammatory cytokines, chemokines and upregulation of co-stimulatory molecules (Compté et al., 2013). In very old frail individuals, this response does not occur to the same extent, and poor nutrition and low muscle mass were closely associated with low activation of this mechanism (Compté et al., 2013; Leng et al., 2007).

Some caution, however, should be taken in the interpretation of these results taking into account the different biological materials used. For instance, some studies have consistently shown that protein markers have higher concentrations in serum than in plasma (Yu et al., 2011). In the case of cytokines, there is also interindividual variability, and higher concentrations are found in female samples (Wong et al., 2008). In the Leng's study cited above, for example, cytokines were evaluated in serum and in our study cytokines were evaluated in plasma. Another factor that can contribute to cytokine variation is the body weight status of the present sample. Our data points to overweight in the subgroup of nonfrail and pre-frail individuals to obesity in the subgroup of the elderly-frail. It is currently accepted that sarcopenia is a likely outcome associated with frailty (Clegg et al., 2013). However, a recent study has shown that fat mass- but not skeletal- muscle mass seems to change in inflammatory profile (Rossi et al., 2019). Hence, more research is warranted in this area. 
In the present study, IL-6 and IL-1 $\beta$ were positively associated with Weakness (low hand grip strength), corroborating the findings previously described by Compté and colleagues (Compté et al., 2013). However, in our adjusted model, IL-1 $\beta$ better explains the Weakness variation (76\%) when compared to IL-6 (26\%). Recent studies suggested that IL-6 also exerts important effects in the activation metabolic and in some anti-inflammatory mechanisms (Maggio et al., 2006). The regression model analysis did show that for each increase of one unit of IL-6, hand grip strength levels decreased on average 0.057 units, proportionally higher than that observed for IL-1 $\beta$ ( 0.005 units). Additionally, our results suggest that an increased pro-inflammatory environment may negatively affect muscle strength levels since the TNF- $\alpha / \mathrm{IL}-10$ ratio was also positively associated with Weakness. For each increase of the TNF- $\alpha / \mathrm{IL}-10$ ratio unit, Weakness levels increased (with $48 \%$ of variance explained in the adjusted model); Slowness increased (with $88 \%$ of variance explained in the adjusted model) and low levels of PA increased (with 15\% of variance explained in the adjusted model).

On the other hand, IL-10 exerts a potent anti-inflammatory activity regulating the immune system response (Bruunsgaard and Pedersen, 2000). A number of studies showed different IL-10 levels in different frail sub-groups, but curiously IL-10 knock out mice have been used as frail-induced animal models (Dagdeviren et al., 2017; von Zglinicki et al., 2016). Decreases in IL-10 have also been linked to the aging process and have been found in obese individuals (Bruunsgaard and Pedersen, 2000). A relationship between IL-10 and Exhaustion emerged in our study and could be explained by the direct relationship between IL-10 and low cardiorespiratory function shown in previous findings (Su et al., 2017). Looking at our results, IL-10 levels were higher in the frail sub-group and elevations in IL-10 are expected as a counter regulatory mechanism to arrest and limit further inflammation in chronically inflamed tissues. Furthermore elevation of IL-10, with or without elevation in IL-6, is associated with an increased risk of cardiovascular disease (Welsh et al., 2011).

Also, TNF- $\alpha$ levels were higher in the pre-frail and frail individuals which could have elicited higher levels of IL-10 in the frail individuals. However, the limited number of studies that associated 
frailty and IL-10 do not permit an accurate inference (von Zglinicki et al., 2016). TNF- $\alpha$ levels were also associated with Exhaustion. According to a recent literature review, increases in the levels of this pro-inflammatory cytokine are associated with the chronic fatigue syndrome, which in turn has a pathophysiological core common to the frail condition with bone loss and muscle weakness (Morey et al., 2016). In our study sample the second highest incidence of comorbidities were bone degenerative diseases (Louati and Berenbaum, 2015). However, our data showed a protective effect of TNF- $\alpha$, specifically, for each unit increase in TNF- $\alpha$ levels, on average, there was a decrease in $0.8 \%$ in the odds ratio of Self-reported exhaustion. This was confirmed by the results found when the regression analysis was applied to the TNF-a/IL-10 ratio, where for each unit increase in this ratio, a decrease of $15 \%$ in the odds ratio of Self-reported exhaustion was also found. At the same time, differences for the TNF- $\alpha /$ IL-10 ratio were also found in the frail sub-groups with a smaller value found for the frail group when compared to the prefrail group. It is possible that a decrease in this ratio in this population, could be the expression of debilitated immune system capacity.

In this study, a slightly but significant increase in $\mathrm{MCH}$ levels in the frail subgroup may give clues to understand an established association between blood cell counts and frailty (Silva et al., 2014). The presence of abnormal red cell indexes suggest the presence of an underlying abnormality before anaemia develops, since high values of $\mathrm{MCH}$ and $\mathrm{MCV}$ are commonly a signal of macrocytic anaemia (Roy, 2011). More common in the elderly, this condition occurs when the blood cells increase in size, often due to vitamin B12 and folic acid deficiency (Silva et al., 2014). Inadequate dietary levels and/or deficient absorption of these vitamins and the use of medication known to impair VitB12 absorption, like metformin and drugs that block stomach acid (Green, 2017), could explain the higher MCH values seen in the frail subgroup (Alves De Rezende et al., 2009). An association between 'inflammaging', frailty and anaemia is also emerging (Röhrig, 2016), linking high levels of pro-inflammatory markers such as IL-1, IL-6 and TNF- $\alpha$ (all increased in our frail subgroup) with anaemia, and this latter one, with functional impairment and frailty ( $\mathrm{Ng}$ et al, 2014). However, until now a cause-and-effect 
relationship between anaemia, frailty, and inflammation has not been established (Leng et al., 2009; Röhrig, 2016).

Symptoms of anaemia include weakness, fatigue and shortness of breath which are also characteristic of exhaustion. Exhaustion is understood as a PF component of fatigue, thus the association between $\mathrm{MCH}$ and Exhaustion found in the present study makes sense and corroborates individual reports of tiredness, discouragement, and low disposition for performance of daily living tasks by frail individuals (Röhrig, 2016). Furthermore, considering the logistic regression model, we s

Found that for each unit increase in $\mathrm{MCH}$, an increase of $26.8 \%$ of reported feelings of Exhaustion occured.

Another important finding of this study was the relationship found between $\alpha$-amylase and Exhaustion, as well as low levels of PA and Weakness (low grip strength). Looking at the results of the regression model, we can see that $\alpha$-amylase better explains the Weakness variation (by 104\%) when compared to others markers, and that for each increase of one unit of $\alpha$-amylase, Weakness increased on average 0.019 units. Moreover, 44\% variation in Low Levels of PA can be explained by $\alpha$-amylase. In the case of Exhaustion, the results of the logistic regression model show that, for each unit increase of $\alpha$-amylase, the feelings of Exhaustion decreased, showing the same protective trend found for TNF- $\alpha$ and the TNF- $\alpha /$ IL-10 ratio.

Research on saliva samples in older populations has grown in the last years for two apparent reasons, which are easy and non-invasive data collection and the existence of moderate and strong correlations with their similar blood markers (Schumacher et al., 2013). Although $\alpha$-amylase was included in our statistical model due to its important contribution to mucosal immunity (acting as a first barrier of host immune defence), in recent years, $\alpha$-amylase has also been studied in a behavioural perspective, due to its identification as a modulator of the autonomic nervous system activity in both biological and psychological stress (Allgrove et al., 2008). The low concentration of $\alpha$-amylase found in the frail subgroup could be explained by the high prevalence of low levels of PA (in $40 \%$ of the 
total sample), since recent findings suggest that maintaining an active life style in older individuals (i.e. moderate regular exercise) can help preserve a reasonable response of the autonomic nervous system (ANS), contributing to a stronger first immune barrier (Walsh et al., 2011).

In contrast to the $\alpha$-amylase results, the levels of salivary COR were higher in the frail subgroup. While COR is a useful maker of hypothalamic-pituitary-adrenocortical activity (HPA) and psychobiological stress, the expression of salivary $\alpha$-amylase occurs in the parotid gland (in response to adrenergic activity) and the sympathetic adrenomedullary system (SAM). This difference may be the first step in understanding the antagonist results found in the frail subgroup. Previous studies have shown that responses of the HPA and the SAM systems may differ depending on the type of stressor (i.e. psychosocial stress, chronic disease, exercise) (Maruyama et al., 2012; Schumacher et al., 2013). As was the case of this study, evidence suggests that higher levels of salivary COR may be due to the vulnerable clinical status of these older women (high CCI values were observed in this sample), corroborated with similar findings from a previous study (Varadhan et al., 2008). Furthermore, experts recognize that high psychological states of depression and anxiety (other important characteristic of this sample) have been empirically linked to dysregulation and inhibition of some immune responses (Morey et al., 2016). This marker also presents an association with the independent PF component of Exhaustion in the adjusted regression model, suggesting associations between global health status, low cardiorespiratory fitness and high levels of biological stress with our data indicating that, for each unit increase in COR values, on average, an increase by $45.2 \%$ in the odds ratio of Exhaustion was found.

Surprisingly, Slowness (low gait speed) and intentional Weight Loss did not correlate with any of the biomarkers analysed. In spite of Slowness being a very apparent trait in frail individuals, studies are unanimous in considering Weakness and the levels of Physical Activity being the most expressive components in FP (Darvin et al., 2014; Furtado et al., 2018). In addition, studies that found associations between different biomarkers, gait speed and weight loss had larger samples, which allowed 
establishment of cut-off values for the low and high concentration of each biomarker, increasing the power of associations (Cable et al., 2018; Mitnitski et al., 2015; Wnuk et al., 2002).

This study has several limitations. First, the number of participants was relatively small, considering the individual variability in some of the biomarkers studied. Future studies with institutionalized samples should include larger samples, especially if involving markers that act in different pathways of the immune system. Second, the cross-sectional data do not permit a causaleffect association. The high number of biomarkers examined was very positive, however, we recognize that when examining the results, it must be taken into account that we introduced in the same statistical model different biological materials (the correlation between the salivary and blood samples of the biological markers used in the present study is, however, well established).

\subsection{Practical applications}

The five components of PF can be positively altered through behavioural changes leading to health improvement. Our study demonstrated that for frail individuals, a significant relationship with important markers of immune and neuroendocrine systems is present. For example, decreasing the pro-inflammatory environment, by implementing adapted exercise programs for institutionalized populations, could improve Strength and Slowness while attenuating the subjective perception of Exhaustion. Pharmacological approaches, including vit.B12, vit.D and acid folic supplementation, could also be envisaged.

\subsection{Conclusion}

The present study indicates that interleukins (IL-10, IL-6 and IL-1 $\beta$, TNF- $\alpha$ and TNF- $\alpha /$ IL10), salivary CORT and $\alpha$-amylase were associated with the frailty status. Salivary $\alpha$-amylase emerged as a novel and important biomarker in the characterization of PF since it was most strongly associated with the physical frailty components. The introduction of salivary biomarkers made the study 
interesting from the methodological point of view, since these biomarkers are intrinsically related to psychological stress and to mucosal immunity in humans. These results also suggest the importance of alterations in the innate immune response in frail individuals, which may make them even more susceptible to pathological agents. The modification of sedentary behaviours and the promotion of an active life style with healthy diets should be advocated as a preventive health policy, aiming to mitigate the effects of frailty to modify biochemical markers related to physical frailty.

\section{Conflicts of interest}

The authors declare that they have no competing interests.

\section{Acknowledgments}

We would like to thank the all residents and workers from SHSC that accepted to participate in this study. Thanks to the students Filipa Pedrosa, Nelba Souza, Fábio Direito, Rafael Carvalho and Taís Rieping for helping with data collection.

\section{Contributors}

Guilherme Furtado drafted the paper. Chupel and Minuzzi helped with data acquisition and biochemical data analysis. Patrício and Loureiro statistically analyzed the data. Hogervorst and Bandelow helped with the proposal, revised the manuscript critically and suggested additional statistical analyses. Teixeira and Ferreira coordinated the research study and revised the manuscript critically. All the authors approved the final version of the manuscript.

\section{Financial support}


This study was financed by the Portuguese National Funding Agency for Science, Research and Technology (FCT), it was integrated as a sub-project in the "PRO-HMESCI: Hormonal mediation of exercise on cognition, stress and immunity" study protocol [FCT PTDC/DTP-DES/0154/2012]. Guilherme Furtado and Matheus Uba Chupel ( $\mathrm{PhD}$ students) were financed by a grant from CAPES/CNPQ - Ministry of Education - Brazil, reference BEX: 11929/13-8 and BEX: 13642/13-8, respectively.

\section{Data Statement}

The directors and managers of all SHSC required confidentiality by the nature of the data collected, and the identification of collaborating SHSC in the previously published study protocol (Teixeira et al., 2016). However, the data can be requested at any time by the reviewers.

\section{References}

Abizanda, P., Romero, L., Sánchez-Jurado, P.M., Martínez-Reig, M., Alfonso-Silguero, S.A., Rodríguez-Mañas, L., 2014. Age, frailty, disability, institutionalization, multimorbidity or comorbidity. Which are the main targets in older adults? The journal of nutrition, health \& aging 18, 622-7. https://doi.org/10.1007/s12603-014-0033-3

Abizanda, P., Romero, L., Sánchez-Jurado, P.M., Martínez-Reig, M., Gómez-Arnedo, L., Alfonso, S.A., 2013. Frailty and mortality, disability and mobility loss in a Spanish cohort of older adults: the FRADEA study. Maturitas 74, 54-60. https://doi.org/10.1016/j.maturitas.2012.09.018

Allgrove, J.E., Gomes, E., Hough, J., Gleeson, M., 2008. Effects of exercise intensity on salivary antimicrobial proteins and markers of stress in active men. Journal of sports sciences 26, 65361. https://doi.org/10.1080/02640410701716790

Alves De Rezende, C.H., Coelho, L.M., Oliveira, L.M., Penha Silva, N., Penha-Silva, N., 2009. Dependence of the geriatric depression scores on age, nutritional status, and haematologic variables in elderly institutionalized patients. Journal of Nutrition, Health and Aging 13, 61721. https://doi.org/10.1007/s12603-009-0172-0

Aw, D., Silva, A.B., Palmer, D.B., 2007. Immunosenescence: Emerging challenges for an ageing population. Immunology. https://doi.org/10.1111/j.1365-2567.2007.02555.X

Batista, M.L., Rosa, J.C., Lopes, R.D., Lira, F.S., Martins, E., Yamashita, A.S., Brum, P.C., Lancha, A.H., Lopes, A.C., Seelaender, M., 2010. Exercise training changes IL-10/TNF- $\alpha$ ratio in the 
skeletal muscle of post-MI rats. Cytokine 49, 102-108.

https://doi.org/10.1016/j.cyto.2009.10.007

Batterham, A.M., Hopkins, W.G., 2006. About Magnitudes. International Journal of Sports Physiology and Performance 1, 50-57.

Baylis, D., Bartlett, D.B., Syddall, H.E., Ntani, G., Gale, C.R., Cooper, C., Lord, J.M., Sayer, A.A., 2013. Immune-endocrine biomarkers as predictors of frailty and mortality: A 10-year longitudinal study in community-dwelling older people. Age 35, 963-971. https://doi.org/10.1007/s11357-012-9396-8

Braga, R., 2013. Ética na publicação de trabalhos científicos. Revista Portuguesa de Medicina Geral e Familiar 29, 354-356.

Bruunsgaard, H., Pedersen, B.K., 2000. Effects of exercise on the immune system in the elderly population. Immunology and Cell Biology 78, 523-531. https://doi.org/10.1111/j.14401711.2000.t01-14-.x

Cable, N., Hiyoshi, A., Kondo, N., Aida, J., Sjöqvist, H., Kondo, K., 2018. Identifying Frail-Related Biomarkers among Community-Dwelling Older Adults in Japan: A Research Example from the Japanese Gerontological Evaluation Study. BioMed Research International 2018, 1-8. https://doi.org/10.1155/2018/5362948

Calvani, R., Marini, F., Cesari, M., Tosato, M., Anker, S.D., Von Haehling, S., Miller, R.R., Bernabei, R., Landi, F., Marzetti, E., 2015. Biomarkers for physical frailty and sarcopenia: State of the science and future developments. Journal of Cachexia, Sarcopenia and Muscle. https://doi.org/10.1002/jcsm.12051

Charlson, M., Szatrowski, T.P., Peterson, J., Gold, J., 1994. Validation of a combined comorbidity index. Journal of clinical epidemiology 47, 1245-51.

Chumlea, W., Baumgartner, N., Baumgartner, N., 1989. Status of anthropometry in elderly subjects3 w composition data. The American Journal of Clinical Nutrition 50.

Chupel, M.U., Direito, F., Furtado, G.E., Minuzzi, L.G., Pedrosa, F.M., Colado, J.C., Ferreira, J.P., Filaire, E., Teixeira, A.M., 2017. Strength training decreases inflammation and increases cognition and physical fitness in older women with cognitive impairment. Frontiers in Physiology 8, 377. https://doi.org/10.3389/fphys.2017.00377

Clegg, A., Young, J., Iliffe, S., Rikkert, M.O., Rockwood, K., 2013. Frailty in elderly people. Lancet 381, 752-62. https://doi.org/10.1016/S0140-6736(12)62167-9

Compté, N., Zouaoui Boudjeltia, K., Vanhaeverbeek, M., De Breucker, S., Tassignon, J., Trelcat, A., Pepersack, T., Goriely, S., 2013. Frailty in Old Age Is Associated with Decreased Interleukin12/23 Production in Response to Toll-Like Receptor Ligation. PLoS ONE 8, e65325. https://doi.org/10.1371/journal.pone.0065325

Conroy, S.P., Stevens, T., Parker, S.G., Gladman, J.R.F., 2011. A systematic review of comprehensive geriatric assessment to improve outcomes for frail older people being rapidly discharged from acute hospital: 'interface geriatrics'. Age and ageing 40, 436-43. https://doi.org/10.1093/ageing/afr060

Dagdeviren, S., Jung, D.Y., Friedline, R.H., Noh, H.L., Kim, J.H., Patel, P.R., Tsitsilianos, N., Inashima, K., Tran, D.A., Hu, X., Loubato, M.M., Craige, S.M., Kwon, J.Y., Lee, K.W., Kim, J.K., 2017. IL-10 prevents aging-associated inflammation and insulin resistance in skeletal muscle. FASEB Journal 31, 701-710. https://doi.org/10.1096/fj.201600832R 
Darvin, K., Randolph, A., Ovalles, S., Halade, D., Breeding, L., Richardson, A., Espinoza, S.E., 2014. Plasma protein biomarkers of the geriatric syndrome of frailty. The journals of gerontology. Series A, Biological sciences and medical sciences 69, 182-6. https://doi.org/10.1093/gerona/glt183

Duarte, M., Paúl, C., 2015. Prevalence of phenotypic frailty during the aging process in a Portuguese community. Revista Brasileira de Geriatria e Gerontologia 18, 871-880. https://doi.org/10.1590/1809-9823.2015.14160

Fernandes, D., 2007. A avaliação das aprendizagens no Sistema Educativo Português. Educação e Pesquisa 33, 581-600. https://doi.org/10.1590/S1517-97022007000300013

Folstein, M.F., Folstein, S.E., McHugh, P.R., 1975. 'Mini-mental state'. A practical method for grading the cognitive state of patients for the clinician. Journal of psychiatric research $12,189-$ 198. https://doi.org/0022-3956(75)90026-6 [pii]

Freitag, S., Schmidt, S., 2016. Psychosocial Correlates of Frailty in Older Adults. Geriatrics 1, 26. https://doi.org/10.3390/geriatrics 1040026

Fried, L.P., Tangen, C.M., Walston, J., Newman, a B., Hirsch, C., Gottdiener, J., Seeman, T., Tracy, R., Kop, W.J., Burke, G., McBurnie, M. a, 2001. Frailty in older adults: evidence for a phenotype. The journals of gerontology. Series A, Biological sciences and medical sciences 56, M146-56.

Furtado, G.E., Caldo, A., Rieping, T.T., Filaire, E., Hogervorst, E., Botelho, A.M.T., Ferreira, J.P., Teixeira, A.M.B., Ferreira, J.P., 2018. Physical Frailty and cognitive status over-60 age populations: a systematic review with meta-analysis. Archives of Gerontology and Geriatrics 78, 240-248. https://doi.org/10.1016/J.ARCHGER.2018.07.004

Gale, C.R., Baylis, D., Cooper, C., Sayer, A.A., 2013. Inflammatory markers and incident frailty in men and women: the English Longitudinal Study of Ageing. Age (Dordrecht, Netherlands) 35, 2493-501. https://doi.org/10.1007/s11357-013-9528-9

González-Vaca, J., de la Rica-Escuín, M., Silva-Iglesias, M., Arjonilla-García, M.D., Varela-Pérez, R., Oliver-Carbonell, J.L., Abizanda, P., 2014. Frailty in INstitutionalized older adults from ALbacete. The FINAL Study: rationale, design, methodology, prevalence and attributes. Maturitas 77, 78-84. https://doi.org/10.1016/j.maturitas.2013.10.005

Guigoz, Y., 2006. The Mini Nutritional Assessment (MNA(registered trademark)) review of the literature - What does it tell us? Journal of Nutrition, Health and Aging 10, 466-485.

Hubbard, R.E., O’Mahony, M.S., Savva, G.M., Calver, B.L., Woodhouse, K.W., 2009. Inflammation and frailty measures in older people. Journal of Cellular and Molecular Medicine 13, 31033109. https://doi.org/10.1111/j.1582-4934.2009.00733.x

Kumar, R., Mohan, N., Upadhyay, A.D., Singh, A.P., Sahu, V., Dwivedi, S., Dey, A.B., Dey, S., 2014. Identification of serum sirtuins as novel noninvasive protein markers for frailty. Aging Cell 13, 975-980. https://doi.org/10.1111/acel.12260

Leng, S.X., Hung, W., Cappola, A.R., Yu, Q., Xue, Q.L., Fried, L.P., 2009. White blood cell counts, insulin-like growth factor-1 levels, and frailty in community-dwelling older women. J Gerontol A Biol Sci Med Sci 64, 499-502. https://doi.org/10.1093/gerona/gln047

Leng, S.X., Tian, X., Matteini, A., Li, H., Hughes, J., Jain, A., Walston, J.D., Fedarko, N.S., 2011. IL-6-independent association of elevated serum neopterin levels with prevalent frailty in community-dwelling older adults. Age and Ageing 40, 475-481. 
https://doi.org/10.1093/ageing/afr047

Leng, S.X., Xue, Q.-L.L., Tian, J., Walston, J.D., Fried, L.P., 2007. Inflammation and frailty in older women. Journal of the American Geriatrics Society 55, 864-871.

https://doi.org/10.1111/j.1532-5415.2007.01186.x

Licastro, F., Candore, G., Lio, D., Porcellini, E., Colonna-Romano, G., Franceschi, C., Caruso, C., 2005. Innate immunity and inflammation in ageing: a key for understanding age-related diseases. Immunity \& ageing : I \& A 2, 8. https://doi.org/10.1186/1742-4933-2-8

Louati, K., Berenbaum, F., 2015. Fatigue in chronic inflammation - a link to pain pathways. Arthritis Research and Therapy. https://doi.org/10.1186/s13075-015-0784-1

Lu, Y., Tze Ying Tan, C., Nyunt, M.S.Z., Hei Mok, E.W., Camous, X., Kared, H., Fulop, T., Feng, L., Pin Ng, T., Larbi, A., 2016. Inflammatory and immune markers associated with physical frailty syndrome: findings from Singapore longitudinal aging studies. Oncotarget 7, 2878328795. https://doi.org/10.18632/oncotarget.8939

Luppa, M., Luck, T., Weyerer, S., König, H.H., Brähler, E., Riedel-Heller, S.G., 2009. Prediction of institutionalization in the elderly. A systematic review. Age and Ageing.

https://doi.org/10.1093/ageing/afp202

Maggio, M., Guralnik, J.M., Longo, D.L., Ferrucci, L., 2006. Interleukin-6 in aging and chronic disease: A magnificent pathway. Journals of Gerontology - Series A Biological Sciences and Medical Sciences. https://doi.org/10.1093/gerona/61.6.575

Maruyama, Y., Kawano, A., Okamoto, S., Ando, T., Ishitobi, Y., Tanaka, Y., Inoue, A., Imanaga, J., Kanehisa, M., Higuma, H., Ninomiya, T., Tsuru, J., Hanada, H., Akiyoshi, J., 2012. Differences in salivary alpha-amylase and cortisol responsiveness following exposure to electrical stimulation versus the Trier Social Stress Tests. PloS one 7, e39375. https://doi.org/10.1371/journal.pone.0039375

Mitnitski, A., Collerton, J., Martin-Ruiz, C., Jagger, C., von Zglinicki, T., Rockwood, K., Kirkwood, T.B.L., 2015. Age-related frailty and its association with biological markers of ageing. BMC medicine 13, 161. https://doi.org/10.1186/s12916-015-0400-x

Morey, J.N., Boggero, I.A., Scott, A.B., Segerstrom, S.C., 2016. Current Directions in Stress and Human Immune Function. Curr Opin Psychol 5, 13-17. https://doi.org/10.1016/j.copsyc.2015.03.007.Current

Ng, T.P., Feng, L., Nyunt, M.S.Z., Larbi, A., Yap, K.B., 2014. Frailty in older persons: Multisystem risk factors and the Frailty Risk Index (FRI). Journal of the American Medical Directors Association 15, 635-642. https://doi.org/10.1016/j.jamda.2014.03.008

Nussenblatt, V., Mukasa, G., Metzger, A., Ndeezi, G., Garrett, E., Semba, R.D., 2001. Anemia and interleukin-10, tumor necrosis factor alpha, and erythropoietin levels among children with acute, uncomplicated Plasmodium falciparum malaria. Clinical and Diagnostic Laboratory Immunology 8, 1164-1170. https://doi.org/10.1128/CDLI.8.6.1164-1170.2001

Petrini, C., 2014. Helsinki 50 years on. La Clinica terapeutica 165, 179-81.

Rockwood, K., Bergman, H., 2012. FRAILTY: A Report from the 3rd Joint Workshop of IAGG/WHO/SFGG, Athens, January 2012. Canadian Geriatrics Journal : CGJ 15, 31-36. https://doi.org/10.5770/cgj.15.35

Röhrig, G., 2016. Anemia in the frail, elderly patient. Clinical Interventions in Aging. 
https://doi.org/10.2147/CIA.S90727

Rossi, F.E., Lira, F.S., Silva, B.S.A., Freire, A.P.C.F., Ramos, E.M.C., Gobbo, L.A., 2019. Influence of skeletal muscle mass and fat mass on the metabolic and inflammatory profile in sarcopenic and non-sarcopenic overfat elderly. Aging Clinical and Experimental Research 31, 629-635. https://doi.org/10.1007/s40520-018-1029-3

Roy, C.N., 2011. Anemia in Frailty. Clinics in Geriatric Medicine. https://doi.org/10.1016/j.cger.2010.08.005

Salimetrics UK, 2017. Salimetrics: Saliva Collection, Saliva EIA kits, Saliva Testing, \& Salivary Bioscience Research [WWW Document]. Salivary Analyts. URL https://www.salimetrics.com/ (accessed 5.28.17).

Schoufour, J.D., Echteld, M.A., Boonstra, A., Groothuismink, Z.M.A., Evenhuis, H.M., 2016. Biochemical measures and frailty in people with intellectual disabilities. Age and Ageing 45, 142-148. https://doi.org/10.1093/ageing/afv152

Schumacher, S., Kirschbaum, C., Fydrich, T., Ströhle, A., 2013. Is salivary alpha-amylase an indicator of autonomic nervous system dysregulations in mental disorders?--a review of preliminary findings and the interactions with cortisol. Psychoneuroendocrinology 38, 729-43. https://doi.org/10.1016/j.psyneuen.2013.02.003

Silva, J.C., de Moraes, Z.V., Silva, C., de Barros Mazon, S., Guariento, M.E., Neri, A.L., Fattori, A., 2014. Understanding red blood cell parameters in the context of the frailty phenotype: Interpretations of the FIBRA (Frailty in Brazilian Seniors) study. Archives of Gerontology and Geriatrics 59, 636-641. https://doi.org/10.1016/j.archger.2014.07.014

Soysal, P., Stubbs, B., Lucato, P., Luchini, C., Solmi, M., Peluso, R., Sergi, G., Isik, A.T., Manzato, E., Maggi, S., Maggio, M., Prina, A.M., Cosco, T.D., Wu, Y.-T.T., Veronese, N., 2016 a. Inflammation and frailty in the elderly: A systematic review and meta-analysis. Ageing Research Reviews 31, 1-8. https://doi.org/10.1016/j.arr.2016.08.006

Soysal, P., Stubbs, B., Lucato, P., Luchini, C., Solmi, M., Peluso, R., Sergi, G., Isik, A.T., Manzato, E., Maggi, S., Maggio, M., Prina, A.M., Cosco, T.D., Wu, Y.T., Veronese, N., 2016b. Inflammation and frailty in the elderly: A systematic review and meta-analysis. Ageing Research Reviews. https://doi.org/10.1016/j.arr.2016.08.006

Su, L., Hao, Q.K., Liu, S., Dong, B.R., 2017. Monocytes Related Inflammatory Biomarkers are Associated With Frailty Syndrome. International Journal of Gerontology 11, 225-229. https://doi.org/10.1016/j.ijge.2017.08.004

Teixeira, A., JP, F., E, H., MF, B., S, B., L, R., A, F., MJ, C., GE, F., MU, C., FM, P., Teixeira, A.M., Ferreira, J.P., Hogervorst, E., Braga, M.F., Bandelow, S., Rama, L., Figueiredo, A., Campos, M.J., Furtado, G.E., Chupel, M.U., Pedrosa, F.M., 2016. Study Protocol on Hormonal Mediation of Exercise on Cognition, Stress and Immunity (PRO-HMECSI): Effects of Different Exercise Programmes in Institutionalized Elders. Frontiers in Public Health 4, 133. https://doi.org/10.3389/fpubh.2016.00133

van der Ploeg, T., Austin, P.C., Steyerberg, E.W., 2014. Modern modelling techniques are data hungry: a simulation study for predicting dichotomous endpoints. BMC Med Res Methodol 14, 137. https://doi.org/10.1186/1471-2288-14-137

Varadhan, R., Walston, J., Cappola, A.R., Carlson, M.C., Wand, G.S., Fried, L.P., 2008. Higher levels and blunted diurnal variation of cortisol in frail older women. J Gerontol A Biol Sci Med 
Sci 63, 190-195. https://doi.org/10.1093/gerona/63.2.190

von Zglinicki, T., Nieto, I.V., Brites, D., Karagianni, N., Ortolano, S., Georgopoulos, S., Cardoso, A.L., Novella, S., Lepperdinger, G., Trendelenburg, A.U., van Os, R., 2016. Frailty in mouse ageing: A conceptual approach. Mechanisms of Ageing and Development.

https://doi.org/10.1016/j.mad.2016.07.004

Walsh, N.P., Gleeson, M.M., Shephard, R.J., Jeffrey, M.G., Woods, A., Bishop, N.C., Fleshner, M., Green, C., Pedersen, K., Hoffman-goetz, L., Rogers, C.J., Gleeson, M.M., Woods, J.A., Bishop, N.C., Fleshner, M., Green, C., Pedersen, B.K., Hoffman-goetz, L., Rogers, C.J., Northoff, H., Abbasi, A., Simon, P., 2011. Part one : Immune function and exercise. Exercise immunology review $17,6-63$.

Walston, J., 2002. Frailty and Activation of the Inflammation and Coagulation Systems With and Without Clinical Comorbidities\&lt;subtitle\&gt;Results From the Cardiovascular Health Study\&lt;/subtitle\&gt; Archives of Internal Medicine 162, 2333.

https://doi.org/10.1001/archinte.162.20.2333

Welsh, P., Murray, H.M., Ford, I., Trompet, S., De Craen, A.J.M., Jukema, J.W., Stott, D.J., McInnes, I.B., Packard, C.J., Westendorp, R.G.J., Sattar, N., 2011. Circulating interleukin-10 and risk of cardiovascular events: A prospective study in the elderly at risk. Arteriosclerosis, Thrombosis, and Vascular Biology 31, 2338-2344. https://doi.org/10.1161/ATVBAHA.111.231795

Wnuk, S.F., Bergolla, L.A., Garcia, P.I., 2002. Studies toward the Synthesis of $\alpha$-Fluorinated Phosphonates via Tin-Mediated Cleavage of $\alpha$-Fluoro- $\alpha$-(pyrimidin-2ylsulfonyl)alkylphosphonates. Intramolecular Cyclization of the $\alpha$-Phosphonyl Radicals. The Journal of Organic Chemistry 67, 3065-3071. https://doi.org/10.1093/gerona/glr099

Wong, H.L., Pfeiffer, R.M., Fears, T.R., Vermeulen, R., Ji, S., Rabkin, C.S., 2008. Reproducibility and correlations of multiplex cytokine levels in asymptomatic persons. Cancer Epidemiology Biomarkers and Prevention 17, 3450-3456. https://doi.org/10.1158/1055-9965.EPI-08-0311

Yao, X., Li, H., Leng, S.X., 2011. Inflammation and immune system alterations in frailty. Clinics in geriatric medicine 27, 79-87. https://doi.org/10.1016/j.cger.2010.08.002

Yu, Z., Kastenmüller, G., He, Y., Belcredi, P., Möller, G., Prehn, C., Mendes, J., Wahl, S., Roemisch-Margl, W., Ceglarek, U., Polonikov, A., Dahmen, N., Prokisch, H., Xie, L., Li, Y., Wichmann, H.E., Peters, A., Kronenberg, F., Suhre, K., Adamski, J., Illig, T., Wang-Sattler, R., 2011. Differences between human plasma and serum metabolite profiles. PLoS ONE 6. https://doi.org/10.1371/journal.pone.0021230 